Reprod. Nutr. Dévelop., 1986, 26 (1 B), 319-320.

\title{
Utilisation comparée des particules colorées et des terres rares pour mesurer la vitesse de passage des résidus alimentaires dans le tube digestif du mouton
}

\author{
J. P. LALLES, C. PONCET
}

Laboratoire de la Digestion des Ruminants, I.N.R.A. Theix, 63122 Ceyrat, France.

Summary. Dyes used as particulate markers provide higher values of mean retention time of feed in the whole digestive tract of sheep than rare earths. The faecal excretion curves of markers five a good estimation of the rumen mean retention time of particles with roughages but not with concentrates.

Les objectifs de cet essai ont été : 1) de comparer les estimations de temps de séjour des résidus alimentaires dans le tube digestif des Ruminants, obtenues par 2 types de marqueurs : colorants (Balch, 1950) et terres rares (Ellis, Matis et Lascano, 1979) ; 2) de comparer les méthodes de calcul des temps de séjour moyens; 3) d'estimer le temps de séjour des résidus dans le rumen directement ou indirectement par analyse mathématique des cinétiques d'excrétion fécale des marqueurs.

Matériel et méthodes. Deux moutons mâles castrés adultes munis de 2 canules (rumen et caillette) ont reçu chacun $1200 \mathrm{~g}$ (8 repas/j) de l'une des 2 rations composées pour moitié de foin haché et d'aliment concentré (son blé ou pulpes de betteraves déshydradées). Les marqueurs, préalablement fixés sur les aliments, selon la technique de Balch (1950) pour les colorants (fuchsine et vert brillant) et selon celle de Teeter, Owens et Mader (1984) pour les terres rares (Ytterbium 169 et Cérium 141) ont été distribués simultanément sous forme d'une dose unique, au début d'un repas.

Résultats et discussion. Le temps de séjour moyen des particules colorées est toujours supérieur à celui des particules marquées par les terres rares (tabl. 1). En effet, le comptage des particules colorées effectué sur la fraction des grosses particules des fèces surestime le temps de séjour alors que la migration des terres rares sur les petites particules a l'effet inverse. L'écart est particulièrement important pour les aliments concentrés. L'index t $80 \%$ (Balch, 1950) fournit toujours les valeurs de temps de séjour les plus élevées, en raison de son caractère arbitraire. Les valeurs obtenues par les paramètres arithmétiques ( $R$ et $t)$ ou par modélisation (TD.T et $\lambda$ TD) sont proches en particulier dans le cas des terres rares, ce qui met en évidence la variabilité du comptage manuel des particules colorées.

Les temps de séjour moyens des aliments dans le réticulo-rumen, estimés par modélisation des courbes de concentration des marqueurs ( $\mathrm{Yb}$ et $\mathrm{Ce}$ ) dans la caillette ou les fèces, approchent de façon satisfaisante la valeur calculée directement 
à partir des prélèvements dans le rumen (tabl. 2), dans le cas du foin mais pas dans le cas des aliments concentrés, pour lesquels les valeurs estimées sont très supérieures.

TABL. 1. - Estimation du temps de séjour dans l'ensemble du tube digestif pour différentes méthodes de calcul.

\begin{tabular}{|c|c|c|c|c|c|c|c|c|c|c|c|}
\hline & & \multicolumn{10}{|c|}{ Temps de séjour dans l'ensemble du dube digestif (heures) } \\
\hline & & \multicolumn{5}{|c|}{ Animal 1 (Foin + Pulpes) } & \multicolumn{5}{|c|}{ Animal 2 (Foin + Son de blé) } \\
\hline & & $\mathrm{t}_{80} \%$ & $\begin{array}{c}R \\
(2)\end{array}$ & $\begin{array}{c}\lambda T D \\
(3)\end{array}$ & $\underset{\langle 4\rangle}{T D . T}$ & $\begin{array}{c}\bar{t} \\
(5)\end{array}$ & $t_{80} \%$ & R & $\lambda T D$ & TD. T & $\bar{t}$ \\
\hline$F^{*}$ & $\begin{array}{l}\mathrm{Ce}^{141} \\
\text { Fuschine }\end{array}$ & $\begin{array}{l}60,5 \\
71,5\end{array}$ & $\begin{array}{l}45,3 \\
55,6\end{array}$ & $\begin{array}{l}47,1 \\
65,9\end{array}$ & $\begin{array}{l}44,8 \\
65,7\end{array}$ & $\begin{array}{l}46,2 \\
59,8\end{array}$ & $\begin{array}{l}38,5 \\
52,0\end{array}$ & $\begin{array}{l}28,4 \\
39,9\end{array}$ & $\begin{array}{l}31,7 \\
42,6\end{array}$ & $\begin{array}{l}30,1 \\
43,4\end{array}$ & $\begin{array}{l}30,6 \\
43,0\end{array}$ \\
\hline A.C. ${ }^{* *}$ & $\begin{array}{l}\text { Yb } b^{169} \\
\text { Vert }\end{array}$ & $\begin{array}{l}61,0 \\
86,5\end{array}$ & $\begin{array}{l}46,1 \\
62,0\end{array}$ & $\begin{array}{l}47,9 \\
77,1\end{array}$ & $\begin{array}{l}46,8 \\
84,5\end{array}$ & $\begin{array}{l}46,9 \\
64,7\end{array}$ & $\begin{array}{l}40,0 \\
82,0\end{array}$ & $\begin{array}{l}29,6 \\
54,6\end{array}$ & $\begin{array}{c}\text { ND } \\
102,4\end{array}$ & $\begin{array}{l}33,3 \\
83,5\end{array}$ & $\begin{array}{l}32,1 \\
57,7\end{array}$ \\
\hline
\end{tabular}

(1) $t_{80} \%=\left(t_{80-5} \%+t_{5} \%\right)$ de Balch $(1950) ;$ (2) $R=$ coefficient de rétention de Castle (1956);

(3) $\lambda \mathrm{TD}=\frac{2}{\lambda_{1}}+\frac{1}{\mathrm{~K}_{2}}+\lambda_{3}$, selon le modèle de Ellis et al. (1979); (4) TD.T $=\frac{2}{\mathrm{~K}_{1}}+\frac{1}{\mathrm{~K}_{2}}+\mathrm{TT}$, selon le modèle de Grovum et Williams (1973); (5) $\bar{t}=$ temps de séjour moyen, selon I hielemans et al. (1978). ${ }^{*} \mathrm{~F}=$ Foin ; ** A.C. Aliment concentré.

TABL. 2. - Comparaison des temps de séjour dans le rumen obtenus par mesure directe à partir des courbes de concentration du marqueur dans le rumen, ou estimés à partir des courbes de concentration du marqueur dans la caillette ou dans les fèces (méthode de calcul de Grovum et Williams, 1973).

\begin{tabular}{lcccccc}
\hline & Temps de séjour dans le rumen (heures) & & & Animal 2 (foin + son) \\
\hline \multicolumn{1}{c}{ Animaux (rations) } & Animal 1 (foin + pulpe) & rumen caillette fèces \\
\hline Lieu de prélèvement & rumen & caillette & fèces & rum \\
\hline foin (cérium 141) & 21,3 & 25,0 & 21,3 & 10,9 & 11,5 & 12,8 \\
concentré (Ytterbium 169) & 18,2 & 20,8 & 24,4 & 11,5 & 10,9 & 18,9 \\
\hline
\end{tabular}

L'emploi préférentiel des terres rares n'exclut pas la prudence requise dans l'estimation du temps de séjour dans le rumen à partir des courbes d'excrétion fécale, dans le cas des aliments concentrés.

Baich C. C., 1950. Factors affecting the utilization of food by dairy cows. 1. - The rate of passagt: of food through the digestive tract. Br. J. Nutr., 4, 361-388.

Castle E. J., 1956. The rate of passage of feedstuffs through the alimentary tract of the goat. 1. - Studies on adult animals fed on hay and concentrate. Br. J. Nutr., 10, 15-23.

Ellis W. C., Matis J. H., Lascano C., 1979. Quantitating the ruminal turnovers. Fed. Proc., 38, 2702-2706.

Grovum W. L., Williams V. J., 1973. Rate of passage of digesta in sheep. 4. - Passage of marker through the alimentary tract and the biological relevance of rate constantes derived from the changes in concentration of marker in faeces. Br. J. Nutr., 30, 313-329.

Teeter R. G., Owens F. N., Mader T. L., 1984. Ytterbium chloride as a marker for particulate matter in the rumen. $J$. anim. Sci., 58, 465-473.

Thielemans M. F., François E., Bodart C., Thewis A., 1978. Mesure du transit gastro-intestinal chez le porc à l'aide de radiolanthanides. Comparaison avec le mouton. Ann. Biol. anim. Bioch. Biophys., 18, 237-247. 